\title{
Preparing for HIV Vaccine Trials in Nigeria: Building the Capacity of the Community and National Coordinating, Regulatory and Ethical Bodies
}

Evaezi Okpokoro ${ }^{1 *}$, Sophia Osawe ${ }^{1,2}$, Pam Datong $^{2}$, Aminu Yakubu $^{3}$, Morenike Ukpong ${ }^{8}$, Paul Orhii $^{4}$, John Idoko ${ }^{5}$, Patrick Dakum $^{1,7}$, Gary Garber $^{6}$ and Alash'le Abimiku' ${ }^{1,2,7}$

${ }^{1}$ Institute of Human Virology, Nigeria

${ }^{2}$ Plateau State Human Virology Research Centre, Jos, Nigeria

${ }^{3}$ National Health Research Ethics Commission, Abuja, Nigeria

${ }^{4}$ National Agency for Food and Drug Administration Control, Abuja, Nigeria

${ }^{5}$ National Agency for the Control of AIDS, Abuja, Nigeria

${ }^{6}$ University of Ottawa/Ottawa Hospital, Canada

${ }^{7}$ Institute of Human Virology, School of Medicine, University of Maryland, Baltimore, USA

${ }^{8}$ New HIV Vaccine Microbicide Advocacy Society, Nigeria

\section{Abstract}

Background: There is paucity of high-level clinical trials in Nigeria for several reasons, which include: lack of trained investigators and mentorship in the implementation of Clinical trials at international standard; inadequate investment and trained regulatory agencies to provide oversight. Therefore, the need for capacity of the national coordinating agencies (NCA); the investigative team, supporting research laboratory and the community to be developed in preparation for future HIV prevention trials including vaccine trials.

Method: In collaboration with the University of Ottawa, three regulatory agencies (NAFDAC -National Agency for Food and Drug Administration Control, NHREC- National Health Research Ethics Committee and NACA-National Agency for Control of AIDS) were identified for capacity building. We developed a phase IIB mock clinical trial model for the purpose of capacity building which created a platform for hands-on training alongside mentoring, meetings, site base visits with full community engagement.

Result: Eight trainees from NACA, NHREC, NAFDAC and the investigative team completed biostatistics and epidemiology course; three trainees attended a Clinical Trial course at the Institute of Human Virology Nigeria and had additional 2-day training/mentoring at the University of Ottawa and thereafter these trainees conducted a 2-day site assessment of the ongoing sero-discordant study in Jos, Nigeria with full community engagement. A trained research team is a member of the national HIV vaccine plan implementation committee and the supporting research laboratory has updated all standard operating manuals for ISO15198 accreditation.

Conclusion: Sustained interaction/integration through trainings/mentorship of National Coordinating Agencies and investigative team promotes commitment relevant for building in-country capacity for successful HIV vaccine clinical trial. An on-going vaccine preparedness HIV sero-discordant couple's cohort is being used to apply principles and concepts of this structure and the trainings in preparation for future HIV prevention including vaccine trials.

Keywords: Vaccine research; Vaccine preparedness; HIV clinical trial; Capacity building

Abbreviations: ARV: Antiretroviral; CAB: Community Advisory Board; CRF: Case Report Form; CSO: Civil Society Organization; GCLP: Good Clinical Laboratory Practice; ICH: International Conference on Harmonization; iPrEx: pre-exposure prophylaxis initiative among men who have sex with men; IRB: Institutional Review Board; NACA: National Agency for Control of AIDS; NAFDAC: National Agency for Food and Drug Administration Control; NHREC: National Health Research Ethics Committee; NHVMAS: New HIV Vaccine Microbicide Advocacy Society; NIAID: National Institute of Allergy and Infectious Diseases; NIH: National Institutes of Health; OHRI: Ottawa Hospital Research Institute; PEPFAR: President Emergency Plan for AIDS Relief; PrEP: pre-exposure prophylaxis; SOP: Standard Operating Procedure

\section{Background}

Over the past 30 years, the number of persons living with HIV has gradually risen to about 34 million persons as at the end of 2011 with over $69 \%$ living in sub-Saharan Africa [1]. Nigeria being the most populous African country with a population of over 140 million [2] is second ( 3 million) to south Africa (5.6 million) in terms of absolute numbers of persons living with HIV [3]. Consequently the advent of President Emergency Plan for AIDS Relief (PEPFAR) in 2004 [4] has significantly impacted positively on the quality and quantity of lives of HIV infected persons. However challenges with access to HIV Counseling and Testing; quality laboratory infrastructure, antiretroviral medications (ARVs) access for all who need it; discrimination and stigmatization; cultural barriers leading to maternal to child transmission; adherence; drug resistance of ARVs; socioeconomic challenges which has diminished the overall impact of the program [5,6]; still remains. It has been established that preventive strategies such as preventive (and perhaps therapeutic) HIV vaccines is necessary to end the epidemic [7]. Recent break through studies such as the partners PrEP study, iPrEx

*Corresponding author: Evaezi Okpokoro, Institute of Human Virology, Nigeria, Tel: +234 8058460765; E-mail: evaezio@yahoo.com; eokpokoro@ihvnigeria.org

Received September 30, 2013; Accepted October 30, 2013; Published November 04, 2013

Citation: Okpokoro E, Osawe S, Datong P, Yakubu A, Ukpong M, et al. (2013) Preparing for HIV Vaccine Trials in Nigeria: Building the Capacity of the Community and National Coordinating, Regulatory and Ethical Bodies. J AIDS Clin Res 4: 260. doi: 10.4172/2155-6113.1000260

Copyright: @ 2013 Okpokoro E, et al. This is an open-access article distributed under the terms of the Creative Commons Attribution License, which permits unrestricted use, distribution, and reproduction in any medium, provided the original author and source are credited. 
(pre-exposure prophylaxis initiative among men who have sex with men) study, microbicides and male circumcision [8-11]; RV144 HIV vaccine phase III trials [12] have been successful in preventing HIV transmission in some of the participants. It is crucial to build on this momentum to develop an effective HIV vaccine that will end the HIV pandemic. Presently, there are several vaccine clinical trials funded by National Institute of Allergy and Infectious Diseases (NIAID) and others for testing at different phases in the United States and a few internationally. Identifying HIV vaccines applicable for developing countries would require phase II or III clinical trial performed in Africa as well. The rationale for HIV vaccine research in Nigeria stems from the argument above and the paucity of high-level clinical trials which have crippled the efforts of African Institutions to effectively contribute to HIV vaccine research. Although there are limited trained investigators and socio-political challenges; by far one of the biggest challenge lies in the inadequate investment in training of national coordinating, ethical and regulatory agencies to adequately review complex clinical trial protocol and monitor the implementation leading to poor supervisory role along with unethical clinical trials culminating in stigmatization and sensitivities from past suboptimal clinical trials $[13,14]$ which has festered mistrust among communities similar to what existed in India [15]. Therefore, the need for a comprehensive and integrated model for building the capacity of national coordinating, ethical and regulatory agencies; and community engagement to support is critical. We have used a mock clinical trial in Nigeria to begin to address this inadequacy and to supplement other HIV vaccine related efforts as done in the past by other investigators $[16,17]$. We document here key elements, efforts, processes, challenges and lessons learnt during our HIV vaccine preparedness for a future HIV vaccine trial.

\section{Methodology}

\section{National agencies involved in HIV coordination, research ethics and vaccine licensure in Nigeria}

Three national agencies in Nigeria have the statutory responsibility for HIV coordination, research ethics and vaccine licensure. The National Agency for Control of AIDS (NACA) is responsible for coordinating the national HIV/AIDS response and advisory role to the Federal Minister of Health. Thus under the coordination of NACA and in conjunction with SHI consulting, Nigeria (a global consultancy firm that aid clients develop and implement actionable strategies) developed a national HIV vaccine plan (http://www.shiconsulting.com/index. php?pageId=10\&cms_showRelease $=1 \& \mathrm{cms}$ release_id=313) which was revised in collaboration with other stakeholders and was launched in September 2012. Some of the key priority areas based on the vaccine plan which our vaccine research strengthens are: "the development of a research literacy team among community stakeholders; the improvement of research skills to conduct HIV vaccine research; improvement of laboratory equipment and personnel needed to conduct baseline studies; synergizing the activities of relevant national agencies responsible for regulating, reviewing and monitoring of all clinical trials; strengthening capacity of national regulatory agencies to approve, monitor and oversee HIV vaccine trials submitted and implemented by researcher" [18]. Second is the National Health Research Ethics Committee (NHREC) which reviews and monitors multi-site complex trials, provides ethical norms and standards for conducting research on humans including clinical trials, registers IRB in the country and audits health research ethics committees and provides guidelines for their functionality. A national code of conduct has been developed with continuous registration and training of ethics committee within the country [19]. Third is the National Agency for Food and Drug Administration Control (NAFDAC) which is empowered to regulate and control the manufacture, importation, exportation, distribution, advertisement, sale and use of food, drugs (including vaccines/biologicals), cosmetics, medical devices, packaged water and chemicals. It is mandated to regulate and control clinical trials to ensure that vaccines/biologicals used in trials are of acceptable quality and efficacy [20]. Several standard operating procedures (SOP) and guidelines have been developed to meet the above mandate such as SOP for obtaining consent, guidelines for clinical trials in Nigeria.

\section{Past efforts}

The Nigerian community has participated in a number of HIV prevention trials, some of which were sub optimal and led to sensitivities and mistrust such as the failed Pfizer trials of 1996 which was reported to lack IRB and NAFDAC approvals and had no documented evidence of informed consent [21,22]. However, prevention trials such as the microbicide study, a phase III double blinded randomized placebo controlled trial, approved by relevant IRBs and NAFDAC was conducted in Lagos and Port-Harcourt between 2004 and 2007 according to good clinical practice as established by the International Conference on Harmonization (ICH) [23]. This improved the regulatory and monitoring oversight function of NAFDAC and our study exposes and trains the regulatory body to an even more complex oversight of clinical trials with experimental vaccines.

\section{Needs assessment for HIV vaccine research}

A need assessment was conducted during four consultative meetings with representatives from NACA, NHREC and NAFDAC. Analyses of existing HIV/AIDS response documents resident in the office of NACA were performed to identify gaps. The summary of the specific capacity building needs (gaps) for an experimental HIV vaccine clinical trials and other biomedical prevention trials are captured in Figure 1: In collaboration with the University of Ottawa and Ottawa Hospital, only national agencies involved in HIV coordination, research ethics and vaccine licensure were identified for training and mentoring. It is expected that these national bodies will step down the trainings to local agencies/IRBs for whom they provide oversight. In addition, we developed a phase IIB mock clinical trial model for the purpose of capacity building to address the identified gaps (Figure 2) which also formed the framework for hands-on training, mentoring, meetings, site base visits and community engagement.

\section{Result}

Over the past 32 months, clearly identified trainings, mentoring, meetings, site base visits were organized towards building the capacity of senior and midlevel officers from the 3 agencies mentioned above. Specific outputs included: number of trainees; application of the training through audit activities and feedbacks; stakeholders meeting attendance; and community activities.

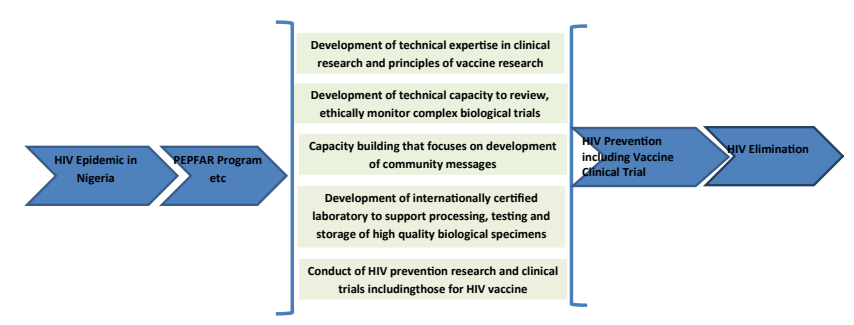

Figure 1: Assessment schematic diagram of identified existing gaps following a needs assessment of national agencies towards HIV vaccine trials. 


\section{Specific trainings and output}

North to South and South to South trainings were successfully carried out to strengthen the technical capacity of government officers of NACA, NHREC and NAFDAC and the research team. Trainings conducted included: online GCLP trainings and on-site research ethics training under the guidance of NHREC. Additional two consecutive short courses on statistical methods and epidemiology funded by NIH Fogarty AIDS training program in collaboration with University of Baltimore, Maryland were organized to fortify basic scientific knowledge on vaccine research. In addition, introductory course on clinical trials organized at the Institute of Human Virology Nigeria were provided to government officers and the research staff. Finally, short course trainings and mentoring in clinical research training were conducted for key officers at the Ottawa Hospital Research Institute (OHRI). Eight trainees from the agencies and the clinical research team attended two weeks intensive certificate course on statistical methods and epidemiology. Thereafter, 17 trainees from among the agencies and the trial team completed the online course on good clinical and laboratory practice as well as an onsite course in research ethics. Three trainees attended the principles of clinical trials followed by clinical research training course at the Ottawa Hospital Research Institute (OHRI), Canada.

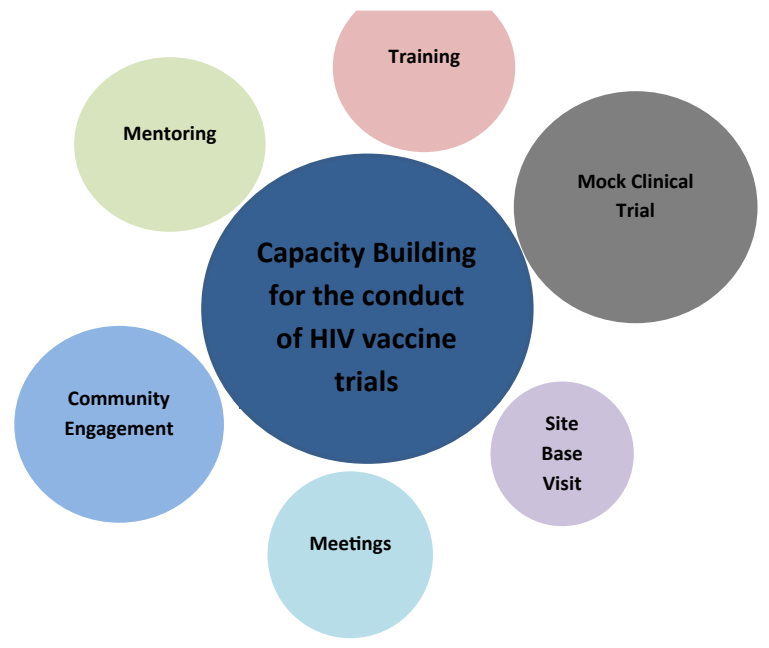

Figure 2: Methods implored to build capacity towards conducting HIV vaccine trials.

\section{Mock clinical trial and site base visits}

A phase IIB mock clinical trial was designed to enroll and follow up 540 HIV negative partners in a sero-discordant relationship. Baseline findings and clinical characteristics among this cohort is published elsewhere. However, following trainings received, officers of collaborating national coordinating agencies were able to successful audit the mock clinical trial using the GCLP report checklist formulated by these agencies. Activities during this audit visit included: review of the regulatory binder; filed informed consent forms; case record forms (CRFs) and other documentations. Other activities included laboratory facility tour to assess standard operating procedures (SOPs); availability of study equipment and maintenance; assessment of storage capacity and space for data entry. At the end of the site visit, these officers provided critical feedback to the clinical research team on compliance with good clinical and laboratory practice (GCLP), the protocol and the principles of the declaration of Helsinki, while reinforcing their oversight function. These schedule site base assessment visits further exposed these assessors to the implementation process and challenges of a vaccine clinical trial in preparation for a future HIV vaccine trial. Some observations and recommendation during the simulated audit are seen in Table 1 .

\section{Community engagement and mobilization}

The New HIV vaccine microbicide advocacy society (NHVMAS), a collaborator on this study with expertise in community engagement focused on public enlightenment and education through regular research literacy programs, workshops, media engagement and advocacy visit using good participatory practice. Community mapping exercise aimed at identifying stakeholders (communities, population and groups, networks and key leaders) for the study was performed. Sixteen civil society organizations (CSO) located within the study site (i.e. Jos, Nigeria) along with fourteen media partners (electronic and print) were identified and supported with scheduled monthly and quarterly research literacy training respectively to ensure community engagement and mobilization. Thus, over 10983 persons (6091 males, 4892 females) within the community were educated on our vaccine research and provided with some research literacy on HIV vaccine clinical trial.

\section{Development of Community Advisory Board (CAB)}

A ten member community advisory board made of representatives of the CSOs, media and other laypersons was formed. They served as a bridge between the community and the research team and initiated

\begin{tabular}{|c|c|c|c|}
\hline & Expectation based on clinical trial GCLP & Audit observation and recommendations & Response by the mock trial team \\
\hline \multirow[t]{2}{*}{$\begin{array}{l}\text { Preliminary } \\
\text { interview }\end{array}$} & $\begin{array}{l}\text { Responsibilities and roles of all members of the trial team } \\
\text { should be clearly written and understood }\end{array}$ & $\begin{array}{l}\text { The research team understood their roles and } \\
\text { responsibility }\end{array}$ & Nil \\
\hline & $\begin{array}{l}\text { A trial team should also consist of pharmacist to manage study } \\
\text { drugs appropriately }\end{array}$ & $\begin{array}{l}\text { The pharmacist was not included in the mock } \\
\text { clinical trial team. }\end{array}$ & $\begin{array}{l}\text { A pharmacist would be identified for } \\
\text { inclusion to the team }\end{array}$ \\
\hline \multirow[t]{2}{*}{$\begin{array}{l}\text { Document } \\
\text { reviews }\end{array}$} & $\begin{array}{l}\text { Study regulatory binders containing the approved version of } \\
\text { the study protocol should be always be situated at study site }\end{array}$ & Regulatory binder was available at the study sites & Nil \\
\hline & $\begin{array}{l}\text { Each page of the case report forms should be signed off by } \\
\text { the principal investigator }\end{array}$ & $\begin{array}{l}\text { Only the last page of the case report forms was } \\
\text { signed off by the principal investigator }\end{array}$ & $\begin{array}{l}\text { Case report forms were re-designed } \\
\text { such each page of the forms contain } \\
\text { a section for the principal investigator } \\
\text { signature }\end{array}$ \\
\hline \multirow[t]{3}{*}{$\begin{array}{l}\text { Facility } \\
\text { inspection }\end{array}$} & $\begin{array}{l}\text { Study rooms should have well secured cabinets to ensure } \\
\text { safety of participant data and ensure confidentiality }\end{array}$ & $\begin{array}{l}\text { There was no secure cabinet in the counselling/ } \\
\text { enrolment room. }\end{array}$ & $\begin{array}{l}\text { A secured cabinet was provided in line } \\
\text { with the recommendation }\end{array}$ \\
\hline & Data storage room should have adequate security measures & $\begin{array}{l}\text { Access into the data storage was not restricted to } \\
\text { only relevant personnel }\end{array}$ & $\begin{array}{l}\text { Additional entry restriction was placed } \\
\text { on the data room thus limiting access. }\end{array}$ \\
\hline & $\begin{array}{l}\text { Prerequisite study and laboratory equipment and SOP should } \\
\text { be available for use }\end{array}$ & SOP for Laboratory equipment were available & Nil \\
\hline
\end{tabular}

Table 1: A Simulated Audit report using NAFDAC Clinical trial GCLP checklist. 
dialogue with the community in support of our vaccine research in their respective constituencies.

\section{Stakeholders meeting}

The study created a unique platform for organized annual meeting chaired by NACA. These meetings of principal officers of these national agencies (i.e. NACA, NAFDAC and NHREC), New HIV Vaccine Microbicides Advocacy Society (NHVMAS), Civil Society Organization members, community advisory board (CAB) representatives, the clinical research team, the media and laboratory personnel created a platform for networking; questions and answers session which heightened understanding and commitment from all present on what it will take to conduct and support HIV vaccine. It also promoted synergy of activities between the national agencies. The meeting identified areas for further discussions and capacity building towards achieving and sustaining the objectives and gains of the study. Representatives of the clinical research team also participated in the revision of the National HIV vaccine planned which was launched in September 2012 (Figure 3).

\section{Discussion}

Much of the about 34 HIV clinical trials in phase I/II that have been completed or are currently taking place in Africa are situated in South and East Africa (i.e. Uganda, Kenya, Botswana, Malawi, Tanzania, South Africa, Rwanda, Zambia, and Mozambique) and Gambia. [24]. Possibly because the prevalence of HIV is highest in these countries, however existing capacities within these countries has been built over time, thus making them more suitable for HIV vaccine research. To establish a similar capacity in West Africa that has a different HIV epidemic of subtype $\mathrm{G}$ and CRF02 rather than the subtype $\mathrm{C}$ that is prevalent in southern Africa; the study reported here strengthened the existing capacity of the national regulatory, ethical and coordinating agencies; and the community in Nigeria to support HIV vaccine trial. While there are other studies in Nigeria that have assessed retention level for HIV vaccine trials [17], this is first documented study that focuses on the holistic preparedness of Nigeria towards HIV vaccine clinical trial similar to the approach used in other countries $[14,15]$ This approach that involves the holistic development of a well-trained clinical, laboratory and data research team alongside a strengthened oversight and regulatory function by coordinating national agencies (NACA, NHREC and NAFDAC); and an informed community provided a possible model for building capacity necessary for a complex biological trial such as the HIV clinical trial.

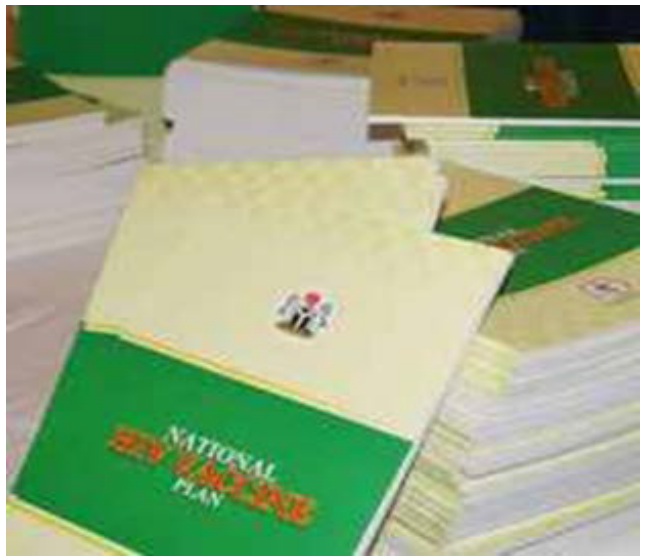

Figure 3: National HIV vaccine plan.

\section{Achievements}

\section{Ability to collaborate with other partners}

Our study strengthened the technical and scientific capacity of officers from national agencies, and led to the inclusion of members of the clinical research team in the revision of the national HIV vaccine plan launched in September 2012 and inclusion in the national vaccine plan implementation committee. Excler and Beyer stressed the importance of such collaborations in overcoming the challenges of implementing a HIV vaccine trial as other AIDS vaccine preparedness studies conducted in India and Uganda reveals [13,15].

\section{Strengthened and louder voice in support of HIV vaccine and other preventive trials in Nigeria}

Through awareness meetings and advocacy of ongoing efforts for a future HIV vaccine trial in Nigeria, stakeholders are more aware and involved in the increased effort to the implementation of the national HIV vaccine plan. Research literacy and advocacy through the involvement of the media is effective in addressing false norms on HIV vaccines trials and bridging trust within the community and opening up discussions on other HIV preventive strategies. In India, a formative study to engage the community was carried 3 years prior to the initiation of its first HIV vaccine clinical trial [15] with good participatory practice $[25,26]$.

\section{Partnerships and collaboration among local CSOs and media}

Involvement of several CSOs by our study has created collateral networks within the community to advocate and educate on HIV vaccine research and trials. This network has identified areas of education and resources for partnerships which is invaluable for the success of research projects. Such additional opportunities for synergy have heightened the drive for preventive trials as recorded by other preparedness studies [15,27].

\section{Development a well characterized cohort for possible HIV vaccine trial}

Baseline data and risk factors of a well characterized sero-discordant population have been gathered for future vaccine trial within same population. This is presented in detailed in other publication by the group. However this cohort presented an opportunity to test procedures outlined in the national HIV vaccine plan and to effectively engaged the national regulatory agencies and the communities in detailed and relevant discussions on future HIV vaccine trials in Nigeria.

\section{Challenges}

Unplanned challenges in getting temporary visa to Canada for short term trainings delayed activities significantly. Some trainees were denied temporary visas following extended visa application process of 2-3 months. This extended visa processing followed by denial in some cases made it impossible to plan trainings for identified potential trainees. Recent unrest by the Boko Haram (a terrorist group) and security challenges at the study sites prolonged the enrollment, delayed site base visits and trainings. These challenges however reflects the reality of conducting research in developing countries and some of the lessons learnt have been useful in strategizing and implementing safely. Since this funding is not guaranteed funding for a vaccine trial, it limits the potential of rolling such preparedness into a full-fledged preventative clinical HIV vaccine trial. However the study team continues to seek for grants that would utilize this well characterized cohort for prevention studies. 


\section{Lessons learnt}

HIV vaccine preparedness have served as a valuable tool in getting countries in resource limited countries ready for the conduct of experimental vaccines or combined biomedical prevention strategies. In order for Nigeria to successfully conduct HIV vaccine in the future, these following lessons would be invaluable:

1. There is need to communicate and strengthen the state arms of the national coordinating agencies for HIV vaccine research. This has the potential to strengthen interactions between the researchers and the government agencies locally and nationally to achieve a common goal.

2. Targeting middle level officers in addition to high level government officers is effective in ensuring the implementation of knowledge acquired and coordination between the different groups.

3. Involvement of relevant high commissions of funding agencies by the host government could reduce visa application delay or rejections for short term and long term trainings in the north.

4. South to South trainings where possible should be explored as this would be cost effective and reduce visa application delays.

5. Finally, local communities are extremely interested in being a credible partner in the effort to find effective HIV vaccines. Involving them very early in this partnership in all fronts is critical.

\section{Conclusion}

Our study has shown that early interaction and integration through meetings, site base visit, trainings and mentorship of national coordinating bodies is critical for building in-country capacity, for successful clinical trials as this creates commitment, promotes ownership, heightened participation and oversight by trained government officials. This is essential for the success of the on-going vaccine preparedness of the cohort of sero-discordant couples by trained research team monitored by trained regulatory and ethics officers. We hope that the building blocks that have been established through this study will lead to the first HIV vaccine clinical trial in Nigeria.

\section{Acknowledgement}

We are grateful to the Canadian Global Health Research Initiative, a research funding partnership made up of three Canadian agencies which includes: the Canadian Institutes of Health Research (CIHR), Canadian International Development Agency (CIDA) and the International Development Research Centre (IDRC) aimed at supporting researchers from low and middle income countries and policy makers as well as bridging the gap [28] for providing funding to strengthen the common platform for HIV vaccine research and HIV care and treatment program in Nigeria.

\section{Reference}

1. UNAIDS (2012) Global Report. Report on the global AIDS epidemic

2. 2006 Population and Housing census.

3. UNAIDS (2012) World AIDS Day Report.

4. US President's Emergency Plan for AIDS Relief (PEPFAR).

5. Abimiku AG, Croxton T, Akintunde E, Okelade B, Jugu J, et al. (2010) Experiences in establishing a PEPFAR-supported laboratory quality system in Nigeria. Am J Clin Pathol 134: 541-549.

6. NACA (2011) Factsheet 2011: Update on the HIVIAIDS Epidemic and Response in Nigeria.

7. Mcnally R (2012) HIV Vaccine Trials: Safe for All. Applied Clinical Trials 21: 50.

8. Baeten JM, Donnell D, Ndase P, Mugo NR, Campbell JD, et al. (2012)
Antiretroviral prophylaxis for HIV prevention in heterosexual men and women. N Engl J Med 367: 399-410.

9. Tobian AA, Serwadda D, Quinn TC, Kigozi G, Gravitt PE, et al. (2009) Male circumcision for the prevention of HSV-2 and HPV infections and syphilis. N Engl J Med 360: 1298-1309.

10. Abdool Karim Q, Abdool Karim SS, Frohlich JA, Grobler AC, Baxter C, et al (2010) Effectiveness and safety of tenofovir gel, an antiretroviral microbicide, for the prevention of HIV infection in women. Science 329: 1168-1174.

11. Grant RM, Lama JR, Anderson PL, McMahan V, Liu AY, et al. (2010) Preexposure chemoprophylaxis for HIV prevention in men who have sex with men. N Engl J Med 363: 2587-2599.

12. Rerks-Ngarm S, Pitisuttithum P, Nitayaphan S, Kaewkungwal J, Chiu J, et al (2009) Vaccination with ALVAC and AIDSVAX to prevent HIV-1 infection in Thailand. N Engl J Med 361: 2209-2220.

13. Mugyenyi PN (2002) HIV vaccines: the Uganda experience. Vaccine 20: 1905 1908.

14. Mugerwa RD, Kaleebu P, Mugyenyi P, Katongole-Mbidde E, Hom DL, et al. (2002) First trial of the HIV-1 vaccine in Africa: Ugandan experience. BMJ 324 226-229.

15. Excler JL, Kochhar S, Kapoor S, Das S, Bahri J, et al. (2008) Preparedness for AIDS vaccine trials in India. Indian J Med Res 127: 531-538.

16. Myles PS, Fletcher HE, Cairo S, Madder H, McRae R, et al. (1999) Randomized trial of informed consent and recruitment for clinical trials in the immediate preoperative period. Anesthesiology 91: 969-978.

17. Edubio A, Agwale S, Bulterys M, Jelpe D, Idoko J, et al. (2010) Preliminary Report on HIV-1 Vaccine Preparedness in Nigeria: Advantages of Recruiting University Students. Viruses 2: 73-77.

18. National HIV Vaccine Plan.

19. National Code for Health Research Ethics.

20. Regulation and Regulatory Affairs Directorate.

21. Stephens J (2006) Panel faults Pfizer in '96 clinical trial in Nigeria. Washington Post .

22. Ezeome ER, Simon C (2010) Ethical problems in conducting research in acute epidemics: the Pfizer meningitis study in Nigeria as an illustration. Dev World Bioeth 10: 1-10.

23. Halpern V, Ogunsola F, Obunge O, Wang CH, Onyejepu N, et al. (2008) Effectiveness of cellulose sulfate vaginal gel for the prevention of HIV infection: results of a Phase III trial in Nigeria. PLoS One 3: e3784.

24. IAVI Report (2013) Database of vaccine candidates in clinical trials.

25. Ruzagira E, Wandiembe S, Bufumbo L, Levin J, Price MA, et al. (2009) Willingness to participate in preventive HIV vaccine trials in a community-based cohort in south western Uganda. Trop Med Int Health 14: 196-203.

26. Dhalla S, Woods R, Strathdee SA, Patrick DM, Hogg RS (2007) HIV vaccine preparedness studies in the organization for economic co-operation and development (OECD) countries. AIDS Care 19: 1118-1127.

27. Vardas E, Buttò S, Glashoff R, Malnati MS, Poli G, et al. (2005) Preparing for phase II/III HIV vaccine trials in Africa. Microbes Infect 7: 1436-1444.

28. IDRC, Global Health Research Initiative. 